
Experiência Acadêmica de Estudantes do Ensino Superior. Consumer Behavior Review, 2(2), 96-107.

ISSN: 2526-7884

Editor: Prof. Dr. Marconi Freitas da Costa Email da revista: cbr@ufpe.br
Avaliação: Double blind review

Recebido: 04 de julho de 2017

Aceito: 01 de maio de 2018

\title{
SATISFAÇÃO COM A EXPERIÊNCIA ACADÊMICA DE ESTUDANTES DO ENSINO SUPERIOR
}

\author{
Bruno Saboya de Aragão \\ Solange Alfinito \\ Correia José Luís
}

Bruno Saboya de Aragão é

Doutorando pelo Programa de PósGraduação em Administração da

Universidade de Brasília - UNB.

Email: brunosaboya@gmail.com.

Solange Alfinito é Professora do

Programa de Pós-Graduação em

Administração da Universidade de

Brasilía - UNB. E-mail:

salfinito@unb.br.

Correia José Luís Graduado em

Administração pela Universidade

de Brasilía - UNB. E-mail:

joseluis03@live.com.pt.

Os autores agradecem aos

avaliadores pelos comentários para melhoria do artigo.

\begin{abstract}
Resumo
O presente estudo tem por objetivo avaliar a satisfação com a experiência acadêmica dos estudantes do ensino superior no curso de administração da Universidade de Brasília, fazendo o comparativo entre os estudantes ingressantes, os que se encontram no meio do curso e aqueles em fase de conclusão. Para tanto, cem alunos de cada etapa do curso participaram do estudo, de caráter quantitativo e descritivo, com coleta de dados realizada presencialmente na Universidade de Brasília, totalizando trezentos estudantes que responderam o questionário sobre satisfação. Os resultados encontrados evidenciam que os estudantes em fase de conclusão estão menos satisfeitos em comparação aos estudantes iniciantes e aqueles que se encontram no meio do curso. Os resultados podem ser utilizados por gestores de instituições de ensino superior, servindo como base para melhorias ao curso, a criação de novas estratégias de ensino e aprendizagem, e melhorias das instalações físicas das IES.

Palavras-chave: Satisfação acadêmica, Experiência acadêmica, Instituições de ensino superior.
\end{abstract}

Esta obra está licenciada com uma Licença Creative Commons Atribuição 4.0 Internacional.

\section{INTRODUÇÃO}

Nos últimos anos, observou-se no Brasil um aumento da população universitária com características bastante heterogêneas, como classe social, gênero, objetivos, expectativas, trajetória acadêmica anterior, faixa etária, dentre outras (Souza, Amaral \& Simões, 2017). Dados do relatório elaborado pelo INEP
(Instituto Nacional de Estudos e Pesquisas Educacionais Anísio Teixeira) em 2016 mostram crescimentos tanto no número de pessoas matriculadas $(2,82 \%$ de 2014 para $2016)$ quanto nas novas vagas abertas $(19,4 \%$ no mesmo período) (INEP, 2016).

0 relatório elaborado pelo INEP também mostra o aumento do número de instituições de 
ensino superior, saltando de 2.368 para 2.407 (INEP, 2016), mostrando consonância entre a demanda e o aumento da oferta. As instituições de ensino superior, portanto,se multiplicaram para atender a essa demanda, no entanto, é preciso que estejam preparadas não só por meio de inovações tecnológicas e novos espaços educativos, mas também com a busca de maior conhecimento sobre o estudante de ensino superior (Barbosa, 2015; Schleich, Polydoro \& Santos, 2006).

Nota-se em alguns países, e também no Brasil, o empenho por parte do governo emabrir mais vagas e instituições de ensino superior (IES) (Pinto, 2004). Porém, falta o mesmo empenho em questões como a adequação das instalações, políticas e processos educativos, além de satisfação do usuário, no caso, os alunos. Tal visão é defendida também por Assis e Gerken (2011), quando os autores versam sobre a fala de importância dada à vida acadêmica em si. Tal fenômeno pode ocorrer por falta de conhecimento sistematizado sobre o estudante universitário brasileiro. Almeida e Soares (2004) constataram fenômeno similar em Portugal, onde existe uma facilidade de acesso ao ensino superior, porém pouca preocupação em que os estudantes sejam bemsucedidos.

Para Almeida, Soares e Ferreira (1999) o sucesso acadêmico não deveser entendido no sentido mais tradicional de desempenho, ou seja, pelas notas obtidas nas avaliações. Deve-se entender o sucesso acadêmico de modo amplo, como toda a experiência vivida pelo estudante no contexto educacional, incluindo seu desempenho cognitivo (raciocínio, conhecimentos, habilidades), afetivo (crenças, valores, atitudes, autoconceito, motivações, satisfação) e social (relações interpessoais).

Sabe-se que, de forma geral, a educação superior provoca mudanças nos estudantes, tanto em nível pessoal, cognitivo, profissional, afetivo e social. A literatura demonstra que os anos que os estudantes frequentam a educação superior são acompanhados por um extenso e integrado conjunto de mudanças decorrentes da diversidade de experiências que compõem este processo educacional, sejam atividades acadêmicas, obrigatórias ou não obrigatórias (Assis \& Gerken, 2011; Astin, 1993; Pascarella \& Terezini, 2005).
Para Astin (1993), o desenvolvimento dos estudantes não é mera consequência do impacto da universidade sobre estes, mas depende do envolvimento deles com os recursos oferecidos pela instituição, além do envolvimento com a própria instituição em si. Para Schleich, Polydoro e Santos (2006), estudiosos buscam compreender melhor o processo de interação entre a instituição e os estudantes e as mudanças provocadas a partir desta interação, construindo instrumentos capazes de caracterizar o estudante e averiguar a experiência vivida durante $\mathrm{o}$ processo de formação.

Assim, a satisfação acadêmica está entre as variáveis analisadas por pesquisadores interessados pela área. Ramos et al. (2015) indicam que estudos sobre esse construto são escassos dada sua importância e apontam para a necessidade de mais estudos sobre o tema, visto a importância da vida acadêmica na vida das pessoas.

A partir do exposto, o objetivo desse trabalho foi avaliar a satisfação com a experiência acadêmica de estudantes do ensino superior em uma universidade pública brasileira. Para tal, foram analisadas três dimensões de satisfação acadêmica, conforme pesquisa e instrumento de Schleich, Polydoro e Santos (2006) de três grupos diferentes de alunos: estudantes de início de curso, estudantes do meio do curso e estudantes de fim de curso (formandos).

\section{SATISFAÇÃO}

Satisfação acadêmica pode ser definida como o nível de satisfação do estudantecom toda a experiência de formação, além de aspectos mais específicos ligados à qualidade do ensino, ao currículo, ao relacionamento com os professores e colegas, à administração, às instalações e recursos da universidade, além da percepção do estudante sobre o ambiente acadêmico e intelectual da instituição (Astin, 1993). Estudar esta satisfação torna-se, portanto, imprescindível para o sucesso de uma IES (Mainardes \& Domingues, 2010). Em suma, a identificação dos fatores que influenciam a satisfação dos estudantes com o curso e com a IES é de importância tanto para fins organizacionais quanto para fins acadêmicos, 
mostrando a importância deste tipo de estudo (Ramos et al., 2015).

Como se vê, a satisfação acadêmica engloba toda trajetória acadêmica. Ela éconsiderada como um processo dinâmico por ser afetada pelas características do estudante e, também por mudar em função da experiência educacional vivida ao longo do curso (Pennington, Zvonkovic \& Wilson, 1989). Abrahamowicz (1988) e Popp et al. (2015) afirmam que as pesquisas realizadas indicamque a percepção dos estudantes quanto à sua satisfação acadêmica interfere no nível de satisfaçãodeste com a instituição, implicando também na decisão de permanecer ou não nessa instituição, tornando o estudo da satisfação dos alunos uma questão relevante também para a própria IES.

Howard e Sheth (1969, p. 145) definem satisfação como o "estado cognitivo de adequação ou inadequação da recompensa pelos sacrifícios efetuados em uma situação de compra". Já Spreng, MacKenzie e Olshavsky (1996) definem satisfação como o grau de uma comparação realizada pelo consumidor, comparando o resultado de determinada transação com uma referência criada ou existente anteriormente, podendo esse resultado ser positivo, neutro ou negativo, gerando, portanto, satisfação positiva ou negativa. Evrard (1995) agrupa alguns tipos de expectativas: experiências anteriores, normas, razão de custos, desejos e valores.

As definições apresentadas, principalmente a de Howard e Sheth (1969), consideram unicamente a satisfação com a transação de produtosOliver (1997) engloba também os serviços em sua definição de satisfação: é o julgamento de que um produto ou serviço, ou parte destes, oferece um nível prazeroso de plenitude relacionado ao consumo. Johnson e Gustafsson (2000) ainda definem a satisfação como cumulativa, um construto abstrato que revela a experiência total de consumo de um bem ou serviço.

Na mesma linha, Gustafsson, Johnson e Roos (2005) afirmam que a satisfação é a comparação entre o desempenho percebido de um produto ou serviço e as expectativas do cliente, e possuem grande influência no processo decisório, podendo culminar em lealdade ou até abandono, se tornando um dos construtos mais importantes do marketing e da prestação de serviços (Medeiros \& Costa, 2016).

Portanto, a satisfação pode ser entendida como um construto multidimensional: ela pode ser influenciada por diversos fatores cognitivos, como valores e normas sociais, tanto quanto por fatores tangíveis, como preço, qualidade do produto ou serviço, entre diversos outros (Zeng et al., 2018). Entende-se, portanto, que o julgamento sobre determinado serviço pode ser influenciado por diversas variáveis simultaneamente, e, consequentemente, a satisfação do consumidor será avaliada a partir desse julgamento realizado. Para a presente pesquisa, esta definição de satisfação será utilizada, com foco na satisfação acadêmica, e o nível desta medido e comparado em relação ao período dos alunos no curso.

Ao passo que a satisfação com serviços prestados é um tema amplamente estudado na literatura sobre consumidores, a satisfação com serviços acadêmicos possui um escopo menor de pesquisas. A satisfação acadêmica, como definido por Schleich, Polydoro e Santos(2006), é um dos construtos estudados para se entender melhor o processo de interação entre os estudantes e as instituições de ensino superior (IES), e, segundo Ramos et al. (2015), o número de estudos sobre o tema é inferior ao necessário, dada a sua importância para a formação dos alunos de universidades.

Conforme exposto anteriormente, a mensuração da satisfação de consumidores abrange diversos construtos. No caso da satisfação acadêmica, as variáveis abrangem toda a experiência de formação, desde aspectos intangíveis, como a qualidade do ensino e o relacionamento com os professores, até aspectos tangíveis, como as instalações da IES e seus recursos (Schleich, Polydoro \& Santos, 2006). Pennington, Zvonkovic e Wilson (1989) consideram a satisfação acadêmica um processo dinâmico, por ser influenciada pela experiência do estudante em relação à vida acadêmica, podendo essa ser modificada por diversos fatores ao longo do tempo.

Schleich, Polydoro e Santos (2006) ainda afirmam que a satisfação acadêmica influencia o nível de envolvimento com a instituição, implicando na decisão de permanecer (ou não) na IES. Tal afirmação mostra a importância de se estudar a satisfação acadêmica, não só para fins acadêmicos, mas também para fins 
organizacionais, visto que a insatisfação leva a maiores níveis de abandono de curso ou até de troca de IES (Strahan \& Credé, 2015).

A mensuração da satisfação dos estudantes, portanto, pode auxiliar na formulação de estratégias e de planejamentos que objetivam a melhoria de currículos e faculdades (Martins, 1998). Para Mainardes e Domingues (2010), identificar os atributos da instituição que fortalecem a satisfação do estudante auxilia as IES a desenvolverem estratégias de aperfeiçoamento dos serviços prestados.

A fim de identificar tais fatores, algumas escalas foram criadas e testadas em ambientes acadêmicos. A primeira relatada na literatura foi a CSSQ - College Student Satisfaction Questionnaire - criado por Betz et al. (1971). Seis dimensões foram retratadas a partir desse instrumento: políticas e procedimentos, condições de trabalho, compensação, qualidade da educação, vida social e reconhecimento. Os autores avaliaram, através de seu levantamento de dados, que a vida social é um fator de grande importância para a vida acadêmica, impactando significativamente a satisfação dos alunos. Essa visão foi reafirmada por DeShields Jr., Kara e Kaynak (2005), que, ao analisar a satisfação de estudantes de graduação, observaram também a experiência como fator determinante de satisfação.

Outro estudo com objetivo similar foi realizado por Martins (1998), denominado Escala de Satisfação Acadêmica. A escala foi composta por cinco dimensões: pertinência das disciplinas, possibilidade de promoção do desenvolvimento, características da docência, características físicas e preparação para o exercício da profissão. Como evidenciado por Sisto et al. (2008), evidências empíricas são escassas sobre esta escala.

Soares, Vasconcelos e Almeida (2002) criaram o Questionário de Satisfação Acadêmica (QSA), que contém três dimensões: social, institucional e curricular. Os resultados da pesquisa elaborada mostraram diferenças entre diversos grupos, como a média das respostas entre as áreas de cursos. As ciências sociaishumanas mostraram a maior média de satisfação nos fatores institucional e curricular. Outro resultado mostrou uma maior satisfação com a instituição de alunos com as maiores notas (Soares, Vasconcelos \& Almeida, 2002).
Os instrumentos listados mostram a multidimensionalidade da variável satisfação acadêmica, e, portanto, a relevância desse construto. A fim também de medir a satisfação de estudantes, Schleich, Polydoro e Santos (2006) criaram, a partir das escalas existentes, a Escala de Satisfação com a Experiência Acadêmica (ESEA), que será utilizada nesse estudo. A escala foi testada em uma IES localizada no estado de São Paulo, e foi a primeira escala a ser desenvolvida e testada no Brasil, e foi testada também no presente trabalho, como relatado na sessão de método. Em estudos posteriores, principalmente a aplicação de Pereira et al. (2018), observou-se uma maior importância, no contexto do ensino superior, dos fatores de satisfação com o curso em detrimento a oportunidades de desenvolvimento. Outro ponto observado nesta pesquisa foi a diferença nas respostas de acordo com a etapa do aluno no curso.

0 trabalho de Ramos et al. (2015) também utilizou a ESEA no contexto da universidade pública nacional, avaliando a relação entre a satisfação acadêmica e a percepção de satisfação acadêmica. Os autores constataram que alunos mais jovens e que realizavam atividades de lazer apresentaram maiores níveis de satisfação com o curso e com a oportunidade de desenvolvimento.

Soares et al. (2014) realizaram um estudo multicultural, abordando o tema de satisfação acadêmica com alunos de universidades públicas e privadas de Brasil e Portugal. Constatou-se, pelo estudo, que os alunos estudados se mostraram satisfeitos com o curso nos três níveis apresentados pela ESEA, sem distinção de país ou área de estudo. Porém, com relação ao tempo do aluno no curso, constatouse que os alunos do sétimo semestre (próximos da conclusão) apresentaram baixos níveis de satisfação. Em ambos os estudos, concluiu-se que a ESEA se mostrou um bom instrumento de mensuração da satisfação acadêmica por parte do aluno (Ramos et al., 2015; Soares et al., 2014).

A ESEA foi escolhida por este estudo, portanto, pelo fato da escala já ter sido utilizada e validada no contexto brasileiro, possibilitando comparações e discussões de evidências, auxiliando na construção de conhecimento com relação à satisfação acadêmica no Brasil. Outro ponto é evidenciado pelos próprios autores 
Schleich, Polydoro e Santos (2006): trata-se de uma escala de fácil compreensão e aplicação, individual ou coletiva, com baixo tempo de resposta, e possibilita a "obtenção de um amplo conjunto de informações sobre a percepção de satisfação do estudante quanto à diversidade de aspectos relacionados à experiência de formação" (Schleich, Polydoro \& Santos, 2006, p. 18).

Antes de apresentar como foi realizada a pesquisa, deve-se fazer um relato do ensino superior no Brasil, a fim de observar seu desenvolvimento e importância para a sociedade como um todo.

\section{ENSINO SUPERIOR NO BRASIL}

Pinto (2004) destaca a política nacional para o setor da educação, que focou na ampliação do número de vagas de ensino superior através do aumento do número de IES privadas a partir da década de 90. Pereira e Gil (2008) também defendem essa visão, destacando que esse aumento se deve à expansão de IES privadas que, por sua vez, focaram em cursos que não requerem alto investimento inicial. Esta expansão pode ser vista em números no relatório de 2016 do INEP, que mostra um aumento no número de instituições (INEP, 2016), mostrando que o aumento de IES é contínuo nos últimos anos.

Medeiros e Rodrigues (2015) resumem o sistema atual de educação superior brasileiro. Para os autores, existe um modelo no qual há instituições públicas e privadas, tendo a primeira o ideal de permanecer com uma qualidade superior às das IES privadas, e estas, por sua vez, preocupadas com o lucro de sócios e acionistas, além do resultado econômico, não possuem necessariamente a educação de qualidade como foco ou objetivo, permanecendo em um segundo patamar, abaixo das IES públicas.

Apesar de destacada melhor performance das IES públicas, houve, a partir da década de 90, um aumento na procura por vagas em instituições privadas, criada pelo aumento considerável na oferta de vagas em IES privadas. Como mostram Nogueira e Oliveira (2015), até a década de 80 as universidades públicas possuíam $50 \%$ das matrículas feitas em IES no Brasil; já em 2010, as IES privadas foram responsáveis por $74,2 \%$ das matrículas realizadas.

De uma forma geral, destaca-se, portanto, um crescimento na oferta de vagas de ensino superior no Brasil a partir da década de 90, criado principalmente pelas políticas públicas que abriram espaço para o crescimento de IES privadas. Para Müller (2005), as transformações das políticas públicas autorizaram também a democratização do acesso ao ensino superior, e incentivaram esse crescimento na oferta de vagas em universidades.

Essa expansão deu-se até o ano de 2008, como destaca Fey, Lucena e Fogaça (2011). Naquele ano, o crescimento de oferta no ensino superior brasileiro foi de $-0,8 \%$, e continua negativo até então. Sendo assim, ainda de acordo com os autores, conforme a capacidade de expansão beira o limite, as IES devem estabelecer ações com objetivo de minimizar a inadimplência e a evasão. Em outras palavras, dado o crescimento até o limite do número de IES privadas e públicas, as instituições existentes devem disputar a concorrência de uma maneira diferente que não a criação de mais vagas.

Dá-se, portanto, a crescente importância da qualidade do ensino nas IES no Brasil. A fim de diminuir a inadimplência, a evasão e até a troca de IES por parte dos alunos, as faculdades e universidades devem focar suas atenções à qualidade interna delas, e não somente à quantidade. De acordo com Fey, Lucena e Fogaça (2011), as IES devem se preocupar também em atender seus alunos da melhor forma possível, devido à grande concorrência existente. Em outras palavras, deve-se dar atenção à qualidade da IES, e o produto final desse investimento deve ser a satisfação do estudante, a fim de minimizar saídas e trocas de universidades.

A partir desse ideal, percebe-se a importância que a satisfação com a experiência em uma IES possui em relação à própria instituição. Como a concorrência atingiu o limite da expansão (Fey, Lucena \& Fogaça, 2011; Bittar, 2008), deve-se atentar, portanto, para o aumento na qualidade da instituição, visando o aumento da satisfação do cliente, no caso, os alunos. A partir disso, a presente pesquisa procurou mensurar a satisfação de alunos do curso de Administração de Empresas da 
Universidade de Brasília, conforme apresentado a seguir.

\section{MÉTODO}

Para atingir o objetivo delimitado para a pesquisa foi desenvolvido um estudo quantitativo com caráter descritivo, a fim de evidenciar essa satisfação. 0 locus da pesquisa foi o curso de Administração na Universidade de Brasília, inaugurado em 1962, apesar de apenas em 1969 foi dado seu reconhecimento, e em 1971 foi estabelecido o currículo pleno do curso. 0 curso tem duração de 4 anos, subdividido em 8 semestres. É o curso com maior número de alunos na universidade, contando-se os dois turnos (diurno e noturno).

\section{Participantes}

O nível de satisfação acadêmica foi medido em três grupos diferentes: estudantes em fase inicial do curso (primeiro e segundo semestres) - Grupo 1; estudantes em meio de curso (terceiro, quarto, quinto e sexto semestres) Grupo 2; e estudantes em fase final de curso, ou formandos (sétimo, oitavo e maiores semestres) - Grupo 3. Os participantes foram escolhidos em uma amostragem não-probabilística por conveniência, onde os pesquisadores aplicaram o instrumento com alunos do curso de administração, pela facilidade de acesso aos alunos, tanto em nível de curso quanto de universidade.

A amostra foi delimitada para que cada grupo contasse com 100 estudantes que responderam à pesquisa, segundo cálculo no software GPower, a fim de se alcançar 95\% de nível de confiança e $5 \%$ de intervalo de confiança, totalizando assim 300 estudantes dos dois turnos do curso. Constatou-se que 49,3\% dos respondentes frequentavam o período noturno, enquanto $50,7 \%$ o período diurno. Além disso, observou-se que $48 \%$ dos respondentes foram do sexo feminino, enquanto $52 \%$ foram do sexo masculino.

\section{Instrumento}

0 instrumento utilizado para medir a satisfação dos estudantes foi a Escala de Satisfação com a Experiência Acadêmica - ESEA - criado e validado por Schleich, Polydoro e Santos (2006). 0 instrumento conta com 35 itens do tipo Likert variando entre totalmente insatisfeito (1) até totalmente satisfeito (5). Os itens são divididos em três dimensões: satisfação com o curso, oportunidade de desenvolvimento e satisfação com a instituição. Para analisar os dados, utilizou-se técnicas de estatística inferencial, visando identificar diferenças nas médias das respostas de cada grupo. Os resultados se encontram a seguir. Conforme posição assumida anteriormente, a ESEA foi escolhida a fim de apresentar melhores comparações com estudos já realizados no contexto brasileiro, e também por já ser um instrumento devidamente aplicado e validado no país.

\section{Procedimentos}

A coleta dos dados foi realizada a partir da aplicação do instrumento citado de maneira presencial aos estudantes do curso de administração, regularmente matriculados na Universidade de Brasília, no período de setembro a outubro de 2015. Para a análise dos dados obtidos, utilizou-se de técnicas estatísticas de comparação de médias, objetivando a comparação dos grupos propostos, assim como de outras variáveis, conforme reportado a seguir.

\section{RESULTADOS}

Primeiramente, uma análise de dados omissos e outliers foi realizada. Como resultado do instrumento ter sido aplicado pessoalmente, não houve respostas excluídas por dados omissos, e também nenhum caso de valores atípicos. Sendo assim, todos as observações obtidas foram utilizadas nas análises que se seguiram.

Procurou-se identificar diferenças de satisfação acadêmica entre os turnos de estudo e o sexo do respondente. Para tal, foram realizados testes de Mann-Whitney $\mathrm{U}$, a fim de verificar se existem diferenças significativas quanto às dimensões de satisfação entre os grupos, conforme indicações de Field (2009). Nenhuma diferença significativa foi identificada entre os sexos feminino e masculino (todas as significâncias foram maiores que 0,05 , segundo orientação de Field (2009). Em outras palavras, os dois grupos estudados apresentaram comportamento homogêneo em relação à satisfação acadêmica. 
Além do sexo do estudante, foram avaliadas diferenças de médias entre os turnos destes, sendo o aluno do turno diurno ou noturno. Da mesma maneira que o sexo, o turno de estudo não mostrou diferenças entre as três dimensões de satisfação acadêmica. Isso quer dizer que, independente de frequentar o turno diurno ou noturno, os alunos não diferem em relação à sua satisfação com a experiência acadêmica, evidenciando também homogeneidade em relação à satisfação.
Em seguida foram conduzidas análises para se comparar a satisfação acadêmica entre os grupos descritos (1, 2 e 3 ). Para tanto, foram realizados diversos testes de Kruskal-Wallis, além de testes post-hoc a fim de identificar as diferenças específicas entre os grupos (Field, 2009). A tabela 1 mostra os resultados desses testes. As três dimensões de satisfação apontaramdiferenças entre as respostas dos grupos. Isso quer dizer que, entre eles, existem diferenças na satisfação acadêmica dos alunos do curso.

Tabela 1

Comparação entre a satisfação acadêmica dos três grupos descritos

\begin{tabular}{|c|c|c|c|c|c|}
\hline Variável & Grupo & $\mathbf{M}$ & DP & $\begin{array}{c}\text { Chi- } \\
\text { quadrado }\end{array}$ & $p$ \\
\hline \multirow{3}{*}{ Satisfação com o curso } & 1 & 3,74 & 0,52 & \multirow{3}{*}{36,27} & \multirow{3}{*}{$0,00^{*}$} \\
\hline & 2 & 3,48 & 0,51 & & \\
\hline & 3 & 3,30 & 0,49 & & \\
\hline \multirow{3}{*}{$\begin{array}{l}\text { Oportunidade de } \\
\text { desenvolvimento }\end{array}$} & 1 & 3,65 & 0,60 & \multirow{3}{*}{55,62} & \multirow{3}{*}{$0,00^{*}$} \\
\hline & 2 & 3,24 & 0,60 & & \\
\hline & 3 & 2,96 & 0,58 & & \\
\hline \multirow{3}{*}{$\begin{array}{l}\text { Satisfação com a } \\
\text { instituição }\end{array}$} & 1 & 3,12 & 0,57 & \multirow{3}{*}{22,68} & \multirow{3}{*}{$0,00^{*}$} \\
\hline & 2 & 3,02 & 0,79 & & \\
\hline & 3 & 2,73 & 0,62 & & \\
\hline
\end{tabular}

Conforme essa diferença é acusada, deve-se realizar testes post-hoc para identificar onde essas diferenças se encontram mais especificamente. Como foram realizados testes com três grupos diferentes, utilizou-se a correção de Bonferroni na análise da significância, a fim de obter resultados mais confiáveis. Pela definição, para os testes posthoc, o nível de significância aceitável foi de 0,0167. A tabela 2 mostra os testes post-hoc realizados, respectivamente para as variáveis satisfação com o curso, oportunidade de desenvolvimento e satisfação com a instituição.

A coluna diferença de média da tabela 4 mostra o resultado da subtração entre o grupo 1 e o grupo 2 de cada análise. Sendo assim, os resultados descritos nesta tabela mostram um decaimento do nível de satisfação entre os grupos. Conforme o teste post-hoc realizado, a maioria das comparações possuem significância, evidenciando que o decaimento da satisfação dos alunos do curso analisado é estatisticamentesignificativo.

Tabela 2

Testes post-hoc realizados para a identificação de diferenças de médias

\begin{tabular}{|c|c|c|c|}
\hline Fator & $\begin{array}{l}\text { Grupos comparados sugiro } \\
\text { usar a numeração deles }\end{array}$ & $\begin{array}{c}\text { Diferença de média } \\
(\mathrm{G} 1-\mathrm{G} 2) \\
\end{array}$ & $P$ \\
\hline \multirow{3}{*}{ Satisfação com o curso } & $\begin{array}{l}\text { Fase inicial } \\
\text { Meio de curso }\end{array}$ & 0,26 & $0,00 *$ \\
\hline & $\begin{array}{l}\text { Fase inicial } \\
\text { Formandos }\end{array}$ & 0,44 & $0,00 *$ \\
\hline & $\begin{array}{l}\text { Meio de curso } \\
\text { Formandos } \\
\end{array}$ & 0,18 & 0,04 \\
\hline \multirow{2}{*}{$\begin{array}{l}\text { Oportunidade de } \\
\text { desenvolvimento }\end{array}$} & $\begin{array}{l}\text { Fase inicial } \\
\text { Meio de curso }\end{array}$ & 0,41 & $0,00 *$ \\
\hline & $\begin{array}{l}\text { Fase inicial } \\
\text { Formandos }\end{array}$ & 0,69 & $0,00 *$ \\
\hline
\end{tabular}




\begin{tabular}{|c|c|c|c|}
\hline Fator & $\begin{array}{l}\text { Grupos comparados sugiro } \\
\text { usar a numeração deles }\end{array}$ & $\begin{array}{l}\text { Diferença de média } \\
(\mathrm{G} 1-\mathrm{G} 2)\end{array}$ & $P$ \\
\hline & $\begin{array}{l}\text { Meio de curso } \\
\text { Formandos }\end{array}$ & 0,28 & $0,00^{*}$ \\
\hline \multirow{3}{*}{$\begin{array}{l}\text { Satisfação com a } \\
\text { instituição }\end{array}$} & $\begin{array}{l}\text { Fase inicial } \\
\text { Meio de curso }\end{array}$ & 0,10 & 0,739 \\
\hline & $\begin{array}{l}\text { Fase inicial } \\
\text { Formandos }\end{array}$ & 0,39 & $0,00^{*}$ \\
\hline & $\begin{array}{l}\text { Meio de curso } \\
\text { Formandos }\end{array}$ & 0,29 & $0,01^{*}$ \\
\hline
\end{tabular}

$\left(^{*}\right) \mathrm{p}<0,0167$, conforme correção de Bonferroni.

Fonte: Elaborado pelos autores.

A partir dos resultados do teste post-hoc e de sua significância estatística, observa-se uma queda nos níveis de satisfação entre os grupos estudados. Essa queda fica mais evidente na figura 1, tornando possível observar esse fenômeno graficamente.

A partir tanto da análise estatística quanto da análise gráfica, evidenciou-se uma diminuição da satisfação acadêmica por parte dos alunos à medida que estes prosseguem no curso. Isso mostra que, ao passo que o estudante adquiri experiências em relação à universidade, o seu nível de satisfação decai, levando-se em conta os resultados obtidos para todas as três variáveis estudadas.

Figura 1 - Análise gráfica damédia da satisfação acadêmica entre os grupos por fator

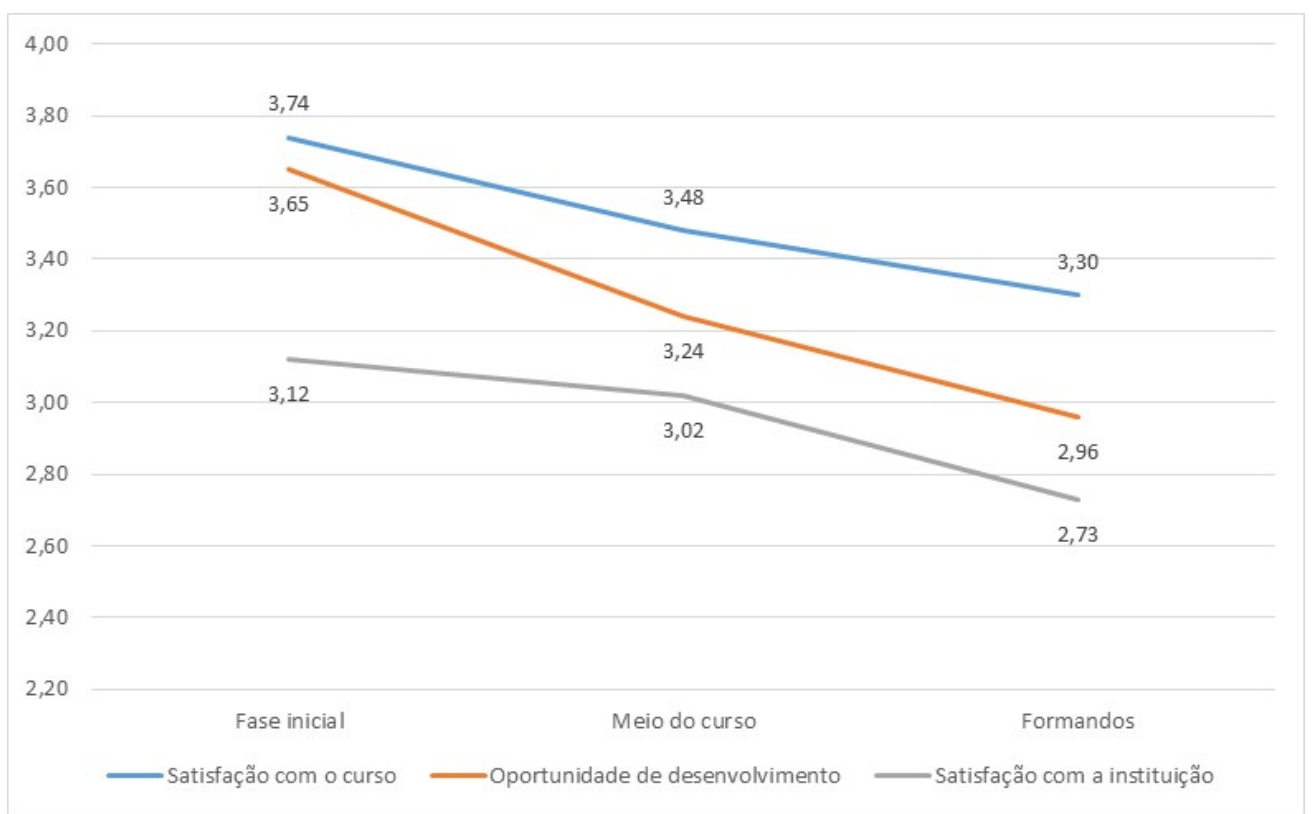

Fonte: Elaborado pelos autores.

Observando-se descritivamente o gráfico, observa-se que os menores níveis de satisfação são relacionados à satisfação com a instituição para os três grupos, ao passo que os maiores níveis de resposta foram identificados com relação a satisfação com o curso. De acordo com Schleich, Polydoro e Santos (2006), a satisfação com a instituição envolve questões relacionadas a infraestrutura, atendimento e instalações da universidade, ao passo que a satisfação com o curso envolve relacionamentos, reconhecimento dos professores e conteúdo em si de aprendizagem.

Observa-se, portanto, que os alunos participantes da pesquisa se mostram menos satisfeitos com a infraestrutura apresentada pela universidade em questão, porém relativamente mais satisfeitos com o curso em 
si, com os professores e o conteúdo ministrado ao longo do curso. A comparação dos resultados encontrados é realizada na seção a seguir.

\section{DISCUSSÃO}

O propósito desse estudo foi avaliar o nível de satisfação dos estudantes do curso de administração de empresas da Universidade de Brasília. Para tal, utilizou-se a Escala de Satisfação com a Experiência Acadêmica (ESEA), criada e validada por Schleich, Polydoro e Santos (2006), a fim de avaliar tal satisfação por três fatores: satisfação com o curso, oportunidade de desenvolvimento e satisfação com a instituição.

Primeiramente, foram analisadas possíveis diferenças de satisfação entre o sexo do estudante e o turno em que frequenta suas aulas. Observou-se que nenhuma dessas duas variáveis apresentaram diferenças de médias em relação à satisfação acadêmica. Tal resultado, em outras palavras, mostra que, independente do sexo ou do turno do aluno, os níveis de satisfação acadêmica são semelhantes. Tal resultado foi encontrado também por Ramos et al. (2015), que estudaram a satisfação no curso de enfermagem em uma universidade pública da região Sul do Brasil, e constataram que, em relação ao sexo do aluno, as respostas foram semelhantes, não configurando uma diferença significativa. Com relação ao turno dos estudantes, não foram encontradas pesquisas com tal análise para fins de comparação.

Com relação à satisfação acadêmica entre os grupos propostos, verificou-se uma tendência significativa de queda dessa variável a medida que os estudantes avançam no curso, ao passo que todas as três variáveis se mostraram decrescentes em relação ao tempo em que o aluno está no curso.

Howard e Sheth (1969) definem satisfação como a percepção da adequação ou inadequação de uma recompensa pelos sacrifícios efetuados. No caso da atual pesquisa, pode-se entender tal sacrifício como todos os esforços presentes na vida acadêmica. Portanto, o aluno espera uma recompensa, seja imediata, como uma boa estrutura da IES, como futura, como uma boa empregabilidade, a partir de todo seu dispêndio de diversos recursos na vida acadêmica.
Analisando os dados e resultados obtidos, observa-se que há uma crescente percepção de inadequação das recompensas esperadas em relação aos sacrifícios prestados. Em outras palavras, à medida que os alunos aumentam seu tempo na universidade, a recompensa recebida passa a ser menos adequada, acarretando em uma diminuição da satisfação do aluno (Howard \& Sheth, 1969). Tal análise também é condizente com a teoria de Johnson e Gustafsson (2000), que definem a satisfação como um atributo acumulativo, ou seja, que absorve diversos fatores ao longo do tempo, entre eles experiência.

Ramos et al. (2015), por outro lado, encontraram que estudantes de semestres mais adiantados possuem uma maior média de satisfação do que estudantes de semestres iniciais. Os autores justificaram esse resultado pelas características do curso de enfermagem: estudantes em estágio final de curso possuem disciplinas relacionadas à prática da profissão, enquanto os estudantes de fase inicial possuem matérias mais teóricas e gerais, dificultando a percepção da aplicação prática de seu conhecimento obtido.

Utilizando novamente a teoria de Howard e Sheth (1969), pode-se entender que os alunos do curso de enfermagem perceberam uma maior recompensa no final do curso, através das disciplinas práticas da profissão. Observando os resultados encontrados relacionados aos alunos de administração de empresas, pode-se entender que os alunos de final de curso não percebem uma inequação das recompensas recebidas, se tornando insatisfeitos de uma maneira geral. Baseando-se no estudo de Ramos et al. (2015), a inclusão de atividades e disciplinas práticas pode fazer com que os alunos de semestres finais possam se sentir mais satisfeitos com a vida acadêmica.

Soares et al. (2014) também avaliaram a satisfação acadêmica de diversos alunos em três universidades de Portugal e do Brasil. No estudo em questão, não se verificou uma tendência crescente ou decrescente clara em relação à idade destes, porém houve uma diferença significativa em relação aos alunos do sétimo semestre, que mostraram satisfação relativamente baixa. Este resultado vai ao encontro dos achados da presente pesquisa. Como os alunos selecionados por Soares et al. (2014) pertenciam aos cursos de marketing, 
finanças e contabilidade, semelhantes à administração, observa-se uma concordância entre os trabalhos.

Comparando-se de uma forma geral os achados desta pesquisa com os trabalhos que utilizaram a ESEA no Brasil, observa-se que alunos de diferentes áreas apresentam diferentes percepções de satisfação, e este entendimento deve ser mais profundamente analisado. As análises mostram uma discrepância entre os níveis de satisfação acadêmica em diversos níveis, como curso e IES. Tal achado abre espaço para outras diversas pesquisas em relação ao tema, e tais recomendações, juntamente com as principais limitações dos estudos, se encontram na sessão a seguir.

\section{CONCLUSÕES E RECOMENDAÇÕES}

0 objetivo dessa pesquisa foi avaliar o nível de satisfação dos estudantes do curso de Administração de Empresas da Universidade de Brasília. Para tal, foi realizada uma pesquisa quantitativa descritiva, na qual, para fim de comparação, dividiu-se os estudantes em três grupos: fase inicial do curso, meio do curso e formandos.

Encontrou-se, após análise inferencial, que as médias de satisfação das três dimensões pesquisadas diminuem em relação ao tempo que o estudante se encontra na universidade. Em outras palavras, conforme o aluno caminha ao longo do curso, menor fica sua satisfação com relação ao curso. Resultados discrepantes foram encontrados na literatura referentes a outros cursos (Ramos et al., 2015) e outros países (Soares et al., 2014). Pesquisas mais profundas sobre o assunto, com abordagens qualitativas, podem ser realizadas para aumentar o entendimento destas diferenças.

A menor satisfação dos estudantes de semestres adiantados pode ser explicada por diversos fatores. Um deles encontra-se na teoria de satisfação. Johnson e Gustafsson (2000) definem a satisfação como cumulativa, um construto abstrato que revela a experiência total de consumo de um serviço. A partir daí, pode-se entender que, à medida que um aluno carrega mais experiência em relação à universidade e ao seu curso, sua satisfação vai diminuindo.
Esse achado possui implicações tanto gerenciais quanto acadêmicas. Tendo tal resultado em mãos, gestores devem atentar não somente para a satisfação dos ingressantes na IES, mas também para os alunos que já estão lá e estão perto de formar-se em seus respectivos cursos. Planejamentos devem ser feitos para que essa satisfação seja alta e linear no decorrer do curso.

Ao ponto de vista acadêmico, deve-se atentar para uma limitação encontrada em respeito à amostra da pesquisa. Foram estudados apenas alunos de um curso de uma universidade, tornando os resultados muito pontuais. Deve-se, portanto, realizar estudos em outros cursos e também em outras universidades, a fim de estabelecer-se comparações entre níveis de satisfação de outros alunos. Novas utilizações da ESEA devem ser aplicadas para que novas comparações e resultados sejam encontrados, objetivando tanto melhorar a satisfação dos alunos de graduação quanto melhorar 0 entendimento do construto em questão.

Propõe-se, portanto, que novas pesquisas sejam realizadas sobre o tema estudado, a fim de se obter maior robustez literária sobre a satisfação acadêmica dos estudantes de IES brasileiras. Conforme exposto, esse tema tornase relevante tanto para acadêmicos, por sua escassez, quanto para gestores, que necessitam manter seus alunos leais à sua IES em um mercado que se tornou altamente competitivo no Brasil ao longo dos anos.

\section{REFERÊNCIAS}

Abrahamowicz, D. (1988). College involvement, perceptions, and satisfaction: A study of membership in student organizations. Journal of College Student Development, 29(3), 233238.

Almeida, L., \& Soares, A. (2004). Os estudantes universitários: sucesso escolar e desenvolvimento psicossocial. Porto: Cabral Editora e Livraria Universitária.

Almeida, L., Soares, A., \& Ferreira, J. (1999). Adaptação, rendimento e desenvolvimento dos estudantes no Ensino Superior: Construção/validação do Questionário de Vivências Académicas. Centro de Estudos em Educação e Psicologia (CEEP), Série Relatórios de Investigação. Braga: Universidade do Minho. 
Assis, D., \& Gerken, C. (2011). Escolhas de vida pósensino médio e representações sociais da univerisade pública. Psicologia em Revista, $17(3), 378-395$.

Astin, A. (1993). What matters in college: Four critical years revisited. 1a ed., São Francisco:JosseyBass.

Barbosa, M. (2015). Expansão, Diversificação, Democratização: questões de pesquisa sobre os rumos do Ensino Superior no Brasil. Caderno CRH, 28(74), 247-253.

Betz, E., Menne, J., Starr, A., \& Klingensmith, J. (1971). Dimensional Analysis of College Student Satisfaction. Measurement and Evaluation in Guidance, 4(2), 99-106.

Bittar, M., Oliveira, J., \& Morosini, M. (2008). Educação Superior no Brasil: 10 Anos Pós-LDB. Brasília. INEP.

Evrard, Y. (1995). A Satisfação dos Consumidores: Situação das Pesquisas. Mimeo PPGA/UFRGS.

Fey, A., Lucena, K., \& Fogaça, V. (2011). Evasão no Ensino Superior: uma pesquisa numa IES do ensino privado. Revista de Humanidades, Tecnologia e Cultura, 1(1), 65-96.

Field, A. (2009). Descobrindo a estatística usando o SPSS. Porto Alegre: Artmed, $2^{\text {a }}$ Ed.

Howard, J.,\&Sheth, J. (1967). A Theory of Buyer Behavior. In: Changing Marketing Systems... Consumer, Corporate and Government Interfaces: Proceedings of the Winter Conference of the Marketing Association, American Marketing Association.

Instituto Nacional de Estudos e Pesquisas Educacionais Anísio Teixeira. Sinopse Estatística da Educação Superior 2016. Brasília: Inep, 2017. Disponível em <http://portal.inep.gov.br/basica-censoescolar-sinopse-sinopse>. Acesso em 09.04.2018.

Johnson, M., \& Gustafsson, A. (2000). Improving customer satisfaction, loyalty, and profit. São Francisco: Jossey-Bass.

Mainardes, E., \& Domingues, M. (2010). Satisfação de estudantes em administração de Joinville/SC. Revista Pensamento Contemporâneo em Administração, 4(2), 7694.

Martins, F. (1998). A satisfação académica: construção de uma escala. In: ALMEIDA, Leandro; GOMES, Maria João; ALBUQUERQUE, Pedro Barbas; CAÍRES, Susana (Edts.). VI Congresso Galaico-Português de Psicopedagogia. Braga: Universidade do Minho.

Medeiros, F., \& Costa, F. (2016). Satisfação de Residentes com seu Lugar: Definição e Proposta de uma Escala de Mensuração de
Múltiplos Itens. Revista Turismo - Visão e Ação - Eletrônica, 18(1), 83-105.

Medeiros, C., \& Rodrigues, H. (2015). O Capital Cultural e a Meritocracia no Acesso ao Sistema de Ensino Superior Brasileiro. Cadernos da Escola de Direito e Relações Internacionais, 1(20), 188-210.

Müller, G. (2005). Fatores que determinam o sucesso de cursos de graduação. Tese de Doutorado. Dissertação (Mestrado em Engenharia de Produção) - Departamento de Engenharia de Produção e Sistemas (EPS), Universidade Federal de Santa Catarina, Florianópolis.

Nogueira, A., \& Oliveira, M. (2015). Mercantilização e relações de trabalho no ensino superior brasileiro. Revista Ciênc. Admin., 21(2), 335364.

Pascarella, E., \& Terenzini, P. (2005). How college affects students. São Francisco: Jossey-Bass.

Pennington, D., Zvonkovic, A., \& Wilson, S. (1989). Changes in college satisfaction across an academic term. Journal of College Student Development, 30(6), 528-535.

Pereira, B., \& Gil, C. (2008). Avaliando a Satisfação de Alunos de Escolas de Administração: uma nova perspectiva de gestão. Revista de Administração da Unimep, 5(1), 1-21.

Pinto, J. (2004). O acesso à educação superior no Brasil. Educação \& Sociedade, 25(88), 727756.

Popp, N., Weight, E., Dwyer, B., Morse, A., \& Baker, A. (2015). Assessing Student Satisfaction Within Sport Management Master's Degree Programs. Sport Management Education Journal, 9(1), 25-38.

Ramos, A., Barlem, J., Lunardi, V., Barlem, E., Silveira, R.,\&Bordignon, S. (2015). Satisfação com a experiência acadêmica entre estudantes de graduação em enfermagem. Texto Contexto Enferm, 24(1), 187-195.

Schleich, A., Polydoro, S., \& Santos, A. (2006). Escala de satisfação com a experiência acadêmica de estudantes do ensino superior. Avaliação Psicológica, 5(1), 11-20.

Sisto, F., Muniz, M., Bartholomeu, D., Pasetto, N., Oliveira, A., \& Lopes, W. (2008). Estudo para a construção de uma escala de satisfação acadêmica para universitários. Avaliação Psicológica, 7(1), 45-55.

Soares, A., Marques, C., Almeida, H., Nascimento, E., Melo, P., Moré, R., \& Gomes, A. (2014). Higher Education and Quality Assessment System: A New Approach Based on Students Perception. Estudos do Isca, 4(10), 1-12.

Soares, A., Vasconcelos, R., \& Almeida, L. (2002). Adaptação e Satisfação na Universidade: Apresentação e validação do Questionário de Satisfação Acadêmica. Contextos e dinâmica da 
vida acadêmica. Guimarães: Universidade do Minho,153-165.

Souza, S., Amaral, S., \& Simões, C. (2017). Políticas Públicas de Educação: 0 Acesso ao Ensino Superior em Questão. Interdisciplinary Scientific Journal, 4(2), 210-225.

Spreng, R., Mackenzie, S., \& Olshavsky, R. (1996). A Reexamination of the Determinants of Consumer Satisfaction. Journal of Marketing, 60(1), 15-32.

Strehen, S.,\&Credé, M. (2015). Satisfaction with College: Re-examining its structure and its relationships with the Intent to Remain in College and Academic Performance. Journal of College Student Retention: Research, Theory \& Practice, 16(4), 537-561.

Zeng, Y., Ling, Y., Huebner, E., He, Y., \& Fu, P. (2018). Assessing the measurement invariance of the Brief Multimensional Students' Life Satisfaction Scale in Chinese and American adolescents. Quality of Life Research, 27(1), 259-266.

\title{
Satisfaction with the Academic Experience of High Education Students
}

\begin{abstract}
The present study had as goal to evaluate the higher education students' satisfaction with the academic experience from the Business School at University of Brasillia, by a comparative between entrants, middle-courses and graduates. Therefore, a hundred students from each group participated in the study, classified as quantitative and descriptive, with a survey conducted in person at the University of Brasilia, totalling three hundred answered questionnaires. The results showed that graduating students are less satisfied with the academic experience than the middle-course students, which are less satisfied than the entrants. The obtained results must be considered and used by higher education institutions, serving as base to review issues related to students' satisfaction, owing to possible improvements in the course itself, the creation of teaching and learning new strategies, and installations improvement.
\end{abstract} Key words: Academic satisfaction, Academic experience, High education institutions. 